\title{
Beware of anal fissures: pemphigus vulgaris of the anal canal
}

\author{
Chara Karatzia, Maria Stefanidou a, Eva Islamidou, ${ }^{a}$ Charikleia Kouvidou ${ }^{b}$, Vrettos Chaniotisc, \\ George Evangelou ${ }^{a}$, Sabine Krueger-Krasagakis ${ }^{a}$, Elias Athanasakis ${ }^{d}$, Konstantin Krasagakis ${ }^{a}$
}

University General Hospital of Heraklion, Crete; Evangelismos General Hospital, Athens, Greece

Abstract

\begin{abstract}
A case of anal pemphigus vulgaris in a 49-year-old female suffering from pemphigus vulgaris of the oral cavity is reported. The oral manifestations were in remission until she presented with episodes of anal pain and bleeding on defecation, initially mimicking anal fissures. Inspection revealed prominent painful erosions in the anal canal with external hemorrhoids and strands of sloughing skin and maceration in the anal verge. Histology and direct immunofluorescence test were consistent with pemphigus vulgaris. The disease was refractory to treatment and complete remission was only achieved with the combination of rituximab and corticosteroids. Anal involvement seems to be an uncommon or underreported manifestation of pemphigus vulgaris. Gastroenterologists should be aware of this entity, especially in areas with a high incidence of the disease, for appropriate diagnosis and management.
\end{abstract}

Keywords Pemphigus vulgaris, anus, erosions, fissures, hemorrhoids

Ann Gastroenterol 2019; 32 (2): 1-3

\section{Introduction}

Pemphigus vulgaris (PV) is a rare autoimmune bullous disease, characterized by the production of pathogenic autoantibodies targeting desmosomal proteins desmoglein 3 and 1 and eliciting epidermal clefting (acantholysis) [1]. A higher incidence of PV has been reported in middle-aged Ashkenazi Jews, Indians, and individuals of Mediterranean and Middle Eastern descent [2].

The clinical phenotype of PV (mucosal, mucocutaneous) is determined by the underlying autoantibody profile. The typical lesions are flaccid blisters and non-healing extensive weeping erosions. The oral cavity is the initial site of involvement in up to $70 \%$ of patients [3] and involvement of other mucous membranes lined by stratified squamous epithelium is possible: nasal, laryngo-esophageal, conjunctival, genital or

Depatment of a Dermatology, University General Hospital of Heraklion, Crete (Chara Karatzi, Maria Stefanidou, Eva Islamidou, George Evangelou, Sabine Krueger-Krasagakis, Konstantin Krasagakis); bPathology, Evangelismos General Hospital, Athens (Charikleia Kouvidou); 'Pathology-Cytology, University General Hospital of Heraklion, Crete (Vrettos Chaniotis); ${ }^{\mathrm{d}}$ General Surgery, University General Hospital of Heraklion, Crete (Elias Athanasakis), Greece

Conflict of Interest: None

Correspondence to: Konstantin Krasagakis, MD, Department of Dermatology, University General Hospital of Heraklion, 71500

Heraklion, Crete, Greece, e-mail: krasagak@med.uoc.gr

Received 15 October 2018; accepted 19 November 2018; published online 20 December 2018

DOI: https://doi.org/10.20524/aog.2018.0340 anal $[4,5]$. Although anal involvement has been suggested in $2-27 \%$ of PV patients $[5,6]$, there is generally little recognition of its importance or experience of this manifestation among physicians, including dermatologists and gastroenterologists. Several cases may initially be misdiagnosed and improperly treated. Since PV, if not treated promptly, usually runs a chronic course and eventually deteriorates, the present case emphasizes the diagnostic challenge of anal PV, initially mistaken for anal fissures.

\section{Case report}

A 49-year-old female patient was referred to our department with painful oral erosions not responding to a previous antiviral therapy given for herpetic stomatitis. Inspection of other mucosal sites revealed mild external hemorrhoids and perianal dermatitis. Her medical history included hypertension, asthma and hemorrhoidal disease. She was never on captopril. Pemphigus vulgaris was diagnosed by means of histology and direct immunofluorescence examination. The patient received systemic therapy with corticosteroids at an initial dose of $0.8 \mathrm{mg} / \mathrm{kg}$ (50 mg per day) and azathioprine $1.5 \mathrm{mg} / \mathrm{kg}$ (100 mg), with marked improvement. The prednisolone dosage was tapered over a period of 8 months to a daily dose of $0.2 \mathrm{mg} / \mathrm{kg}(12.5 \mathrm{mg})$ combined with azathioprine $1.5 \mathrm{mg} / \mathrm{kg}$. At that time she suffered from recurrent episodes of anal pain and bleeding on defecation. After surgical evaluation the diagnosis of anal fissures was made and proctoscopy with sphincterotomy was performed. However, her complaints worsened, with the anal 
pain aggravating by long sitting or defecation, forcing her to delay or avoid defecation. These complaints caused a major impairment in her quality of life. When she was referred to our department again, a thickened and edematous anal orifice was seen, with irregular rings and everted mucosal surface. Three painful linear erosions located in the 6, 9 and 12 o'clock position were observed, mimicking anal fissures (Fig. 1A); in contrast, real anal fissures are usually located posteriorly in the midline. The anal skin had a whitish macerated appearance with strands of sloughing skin. Careful inspection of the anal verge, by placing a lubricated finger against the anus and asking the patient to bear down to relax the external sphincter, allowed gentle protrusion of distal anoderm and revealed prominent erosions (Fig. 1B). Anoscopy revealed circular involvement of pemphigus of the anus below the dentate line.

Vaginal, ophthalmologic and laryngoscopic examinations showed no PV involvement. Histological examination of a biopsy from the anus showed suprabasal blister formation and acantholysis of keratinocytes (Fig. 2A). Direct immunofluorescence from the perilesional area revealed granular deposits of IgG and C3 in the intracellular spaces of epidermis (Fig. 2B). An autoantibody titer against desmoglein 3 in the patient's serum was not determined. Once the diagnosis of anal PV was established, oral prednisolone was increased to $0.5 \mathrm{mg} / \mathrm{kg}$ (35 mg per day) and azathioprine $2.3 \mathrm{mg} / \mathrm{kg}$ (150 mg/day), with partial improvement in 4 weeks and slow tapering of steroids. Disease exacerbations with painful erosions of the anal canal were treated with an increase of steroids while on azathioprine $150 \mathrm{mg} /$ day. One year later, azathioprine was replaced by mycophenolate mofetil, given that the disease had not been cleared with prednisolone remaining always over $20 \mathrm{mg} /$ day. Fourteen months later, $20 \mathrm{mg} /$ week methotrexate was added and mycophenolate mofetil was gradually tapered and finally stopped, since the anal erosions did not completely heal and prednisolone was never below $15 \mathrm{mg} /$ day. She finally received rituximab in 4 weekly infusions of $375 \mathrm{mg} / \mathrm{cm}^{2}$ and complete remission was achieved within 2 months, with prednisolone being tapered in $2.5 \mathrm{mg}$ steps every 2 months. The patient has now been completely clear for 5 months with only prednisolone $5 \mathrm{mg} /$ day. The patient was also instructed to consume food with high fiber content and fluid intake and to preserve good hygiene after defecation.

\section{Discussion}

Although PV is known to involve the anal area, the frequency at which it occurs varies widely among studies, ranging from 2-27.9\%. Chams-Davatchi et al [5] observed anal lesions in $2 \%$ of 1209 patients with PV, while Malik et al [7] reported an incidence of $10 \%$ in their retrospective cohort of $160 \mathrm{PV}$ patients. Kherzi et al [6], in a prospective study of 168 newly diagnosed cases of PV, found a $27.9 \%$ rate of anal involvement and suggested that anal PV seems underreported. Anal involvement was significantly associated with PV in other mucosal sites (ophthalmic, nasal, genital). It was also
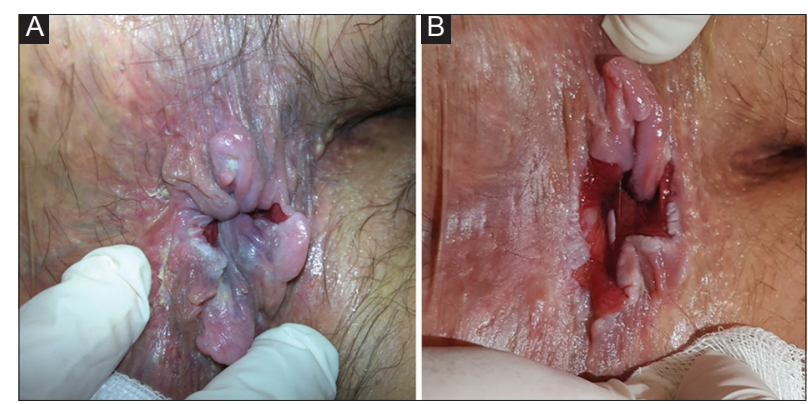

Figure 1 Three painful linear erosions located in the 6,9 and 120 ' clock position were observed, mimicking anal fissures. The anal skin had a whitish macerated appearance with strands of sloughing skin (A) Inspection of the distal anoderm revealed circular erosions of the anus (B)
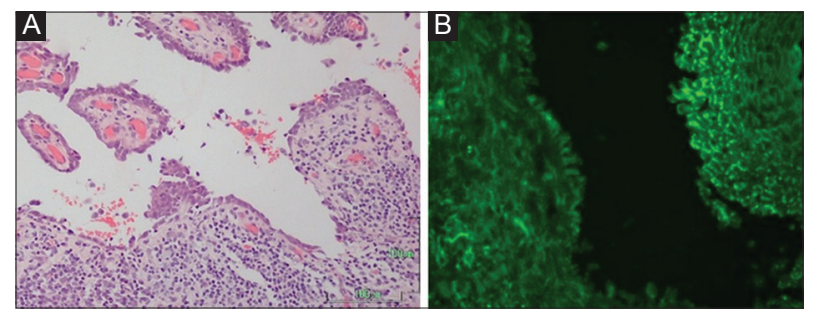

Figure 2 Histological examination of a biopsy from the anus, showing formation of suprabasal blister and acantholysis of keratinocytes (A). Direct immunofluorescence from the perilesional area revealed granular deposits of IgG in the intracellular spaces of epidermis (B)

significantly correlated with the severity of concomitant oral PV, but not with the severity of cutaneous lesions [6]. Hemorrhoids were observed in $17(10 \%)$ patients; interestingly, 16 of the 17 patients with hemorrhoids also had anal PV, suggesting that repeated trauma during defecation might be a triggering factor. Our case illustrates that anal mucosa is a site of PV recurrence, even when other sites are in remission.

PV treatment challenges include shorter treatment periods and better quality of life for the patients [8]. Since early initiation of treatment, before PV becomes too severe or widespread, has been associated with improved prognosis in general [9], early recognition of anal involvement may be important in the management of PV patients. Regarding therapy, our patient responded favorably to treatment with rituximab, in concordance with the results of a previous study with this drug in PV patients of the same genetic heritage [10].

Routine anal clinical examination is recommended even in asymptomatic patients with PV, while anoscopy performed by a colorectal specialist is indicated in patients with local discomfort and/or unexplained anal pain, particularly in the presence of hemorrhoids. Confirmatory skin biopsy and direct immunofluorescence testing are essential in order to achieve prompt diagnosis and appropriate management of PV. Gastroenterologists should be aware of this manifestation and have a high index of suspicion for PV alongside more common differential diagnoses, such as anal fissures, hemorrhoids or cancer, especially in patients with either a history of pemphigus or a not yet diagnosed oral erosive disease. 


\section{References}

1. Kasperkiewicz M, Ellebrecht CT, Takahashi $\mathrm{H}$ et al. Pemphigus. Nat Rev Dis Primers 2017;3:17026.

2. Bystryn JC, Rudolph JL. Pemphigus. Lancet 2005;366:61-73.

3. Firth N, Rich A, Varigos G, Reade PC. Oral pemphigus vulgaris in young adults. Int J Dermatol 1991;30:352-356.

4. Palleschi GM, Cipollini EM, Lotti T. Development of oesophageal involvement in a subject with pemphigus vulgaris: a case report and review of the literature. J Eur Acad Dermatol Venereol 2002;16:405-408.

5. Chams-Davatchi C, Valikhani M, Daneshpazhooh M, et al. Pemphigus: analysis of 1209 cases. Int J Dermatol 2005;44:470-476.
6. Khezri S, Mahmoudi HR, Masoom SN, et al. Anal involvement in pemphigus vulgaris. Autoimmune Dis 2013;2013:609181.

7. Malik M, El Tal AE, Ahmed AR. Anal involvement in pemphigus vulgaris. Dis Colon Rectum 2006;49:500-506.

8. Gregoriou S, Efthymiou O, Stefanaki C, Rigopoulos D. Management of pemphigus vulgaris: challenges and solutions. Clin Cosmet Investig Dermatol 2015;8:521-527.

9. Cholera M, Chainani-Wu N. Management of pemphigus vulgaris. Adv Ther 2016;33:910-958.

10. Gregoriou S, Giatrakou S, Theodoropoulos K, et al. Pilot study of 19 patients with severe pemphigus: prophylactic treatment with rituximab does not appear to be beneficial. Dermatology 2014;228:158-165. 